\title{
A Note on Acyclic Coloring of Central Graphs
}

\author{
Dr. K. Thilagavathi \\ Associate Professor \\ Department of mathematics \\ Kongunadu Arts and Science College Coimbatore \\ Tamilnadu-641029, India.
}

\author{
Mr. P. Shanas Babu \\ Research Scholar \\ Department of mathematics \\ Kongunadu Arts and Science College Coimbatore \\ Tamilnadu-641029, India.
}

\begin{abstract}
In this paper, we discuss the acyclic vertex colouring and acyclic chromatic number of central graph of Dutch-windmill graph and Sunlet graph and acyclic colouring of some other graphs.
\end{abstract}

\section{General Terms}

Central graph of Dutch-windmill graph and Sunlet graph are denoted by $C\left(D_{3}{ }^{m}\right)$ and $C\left(S_{n}\right)$ respectively. The Crown graph is denoted by $S_{n}^{0}$

\section{Keywords}

Central graph, Dutch-windmill graph, Sunlet graph, Crown graph, acyclic colouring, acyclic chromatic number.

\section{INTRODUCTION}

Let $G$ be a finite undirected graph with no loops and multiple edges. The central graph [17] of a graph $G, C(G)$ is obtained by subdividing each edge of $G$ exactly once and joining all the nonadjacent vertices of $G$. By the definition $p_{c G}=p+q$. For any

$p, q$ graph there exist exactly $p$ vertices of degree $p-1$ and $q$ vertices of degree 2 in $C(G)$.

The Dutch-Windmill graph $[11,14]$, denoted $D_{3}{ }^{m}$, also called a friendship graph, is a graph obtained by taking $m$-copies of the cycle $C_{3}$ with a vertex in common.

The $n$-sunlet graph $S_{n}$ [12] is the graph on $2 n$ vertices obtained by attaching $n$ pendent edges to the cycle graph $C_{n}$.

The Crown graph $S_{n}^{0}$ [5] for an integer $n>2$ is the graph with the vertex set $\left\{u_{1}, u_{2}, u_{3} \ldots u_{n}, v_{1}, v_{2}, v_{3}, \ldots v_{n}\right\}$ and edge set $\left\{\left(u_{i}, v_{i}\right): 1 \leq i, j \leq n, i \neq j\right\}$.

A proper vertex colouring of a graph is acyclic if every cycle uses at least three colours [13].The acyclic chromatic number of $G$, denoted by $a G$, is the minimum $k$ such that $G$ admits an acyclic $k$-colouring.

\section{ACYCLIC COLOURING OF $C\left(D_{3}{ }^{m}\right)$}

\subsection{Theorem}

For the Dutch-windmill graph $D_{3}{ }^{m}, a\left[C\left(D_{3}{ }^{m}\right)\right]=2 m=\Delta$ where $\Delta$ is the maximum degree of $G$.

\section{Proof}

Consider the Dutch-Windmill graph $D_{3}{ }^{m}$ formed by $m$-copies of the cycle $C_{3}$ with vertices $v_{1}{ }^{i}, v_{2}{ }^{i}, v_{3}^{i}, i=1,2,3, \ldots . m$, named in counter clockwise direction in which $v_{1}^{1}=v_{1}^{2}=v_{1}^{3}=\ldots=v_{1}^{m}$ is the common vertex. Let $v_{h, k}{ }^{i}$ represents the newly introduced vertex in the edge connecting $v_{h}{ }^{i}$ and $\quad v_{k}{ }^{i}$ for $i=1,2 \ldots m$ and $x, y=1,2,3$. Now assign a proper colouring to these vertices as follows. Consider a colour class $C=c_{1}, c_{2}, c_{3}, \ldots . c_{2 m}$ and let the pair $c_{h} c_{k}$ represents an edge whose end points are coloured as $c_{h}$ and $c_{k}$. Assign the color $c_{1}$ to the common vertex $v_{1}{ }^{i}, i=1,2, . . m$ and the color $c_{2 k-1}$ to the vertex $v_{3}{ }^{k}$ for $k=1,2, \ldots m$. Next assign the colour $c_{2 k}$ to the vertex $v_{2}^{k+1}$ for $k=2,3,4 \ldots m-1$ if $m>2$ and $c_{2 m}, c_{3}$ to the vertices $v_{2}{ }^{1}$ and $v_{2}{ }^{2}$ respectively. Such a colouring excludes the newly introduced vertices. Assign the colour $c_{2}$ to the newly introduced $3 m$ vertices $v_{h, k}{ }^{i}$ for $i=1,2,3, . . m$ and $x, y=1,2,3$. Now we prove that the above said colouring is acyclic. That is it does not contain any bichromatic cycles. It is obvious that to form a 2chromatic cycle both colours should occur at least twice. So in the above said colouring, the color classes $c_{k}, 4 \leq k \leq 2 m$ never induce a 2-chromatic cycle. Now we need to examine the subgraphs induced by $\left\langle c_{i}, c_{j}\right\rangle$ for $i=1,2$ and $j=2,3$ with $i \neq j$ whether they induce a 2-chromatic cycle or not.

Case 1. If $i=1$ and $j=2$, then subgraph induced by $\left\langle c_{1}, c_{2}\right\rangle$ is the union of a path tree with $\Delta-2$ pendent vertices and $m-1$ isolated vertices, which is clearly a forest.

Case 2. If $i=1$ and $j=3$, then subgraph induced by $\left\langle c_{1}, c_{3}\right\rangle$ is the union of a path $P_{2}$ and a single isolated vertex, which is a forest. 
Case 3. If $i=2$ and $j=3$, then subgraph induced by $\left\langle c_{2}, c_{3}\right\rangle$ is the union of a path $P_{4}$ and $3(m-1)$ isolated vertices, which also forms a forest.

OR In each case we can easily verify the result $\varepsilon=v-\omega$, which is the necessary and sufficient condition for a forest.

Thus any pair of the colour class will never induce a 2-

chromatic cycle in the graph. By the very construction the colouring is minimum.

Therefore, $a\left[C\left(D_{3}{ }^{m}\right)\right]=2 m=\Delta$.

\section{Example}

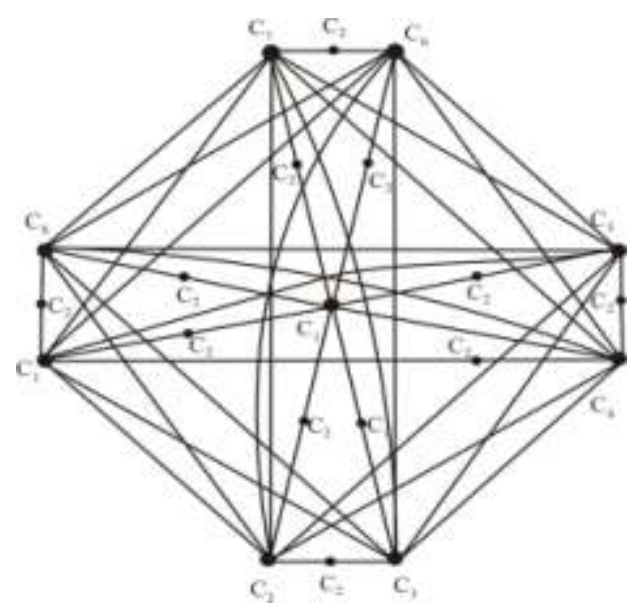

Figure 1

$$
a\left[C\left(D_{3}^{4}\right)\right]=8
$$

\section{ACYCLIC COLOURING OF $C\left(S_{n}\right)$}

\subsection{Theorem}

For the sunlet graph $S_{n}$, the acyclic chromatic number $a\left[C\left(S_{n}\right)\right]=2 n-2$

\section{Proof}

Let $S_{n}$ be the the $n$-sunlet graph having $2 n$ vertices formed by attaching $n$ pendent edges to the cycle graph $C_{n}$. Let $V\left(C_{n}\right)=\left\{u_{1}, u_{2}, u_{3} \ldots u_{n}\right\}$. The pendent edge connected to the vertex $u_{i}$ be $u_{i} v_{i}, i=1,2,3 \ldots n$. That is $v_{1}, v_{2}, v_{3} \ldots v_{n}$ are the pendent vertices. Now consider $C\left(S_{n}\right)$, let the vertex introduced in the edge joining $u_{i}$ and $u_{j}$ be $u_{i, j}, \quad i=1,2,3 . . . n$, $j=2,3,4 \ldots n$ and vertex in the edge joining $u_{i}$ and $v_{j}$ be $v_{i j}$. Now in $C\left(S_{n}\right),\left[v_{1}, v_{2}, v_{3} \ldots v_{n}\right]$ is a complete graph on $n$ vertices.
Assign a colouring to $C\left(S_{n}\right)$ as follows. Colour the vertex $v_{i}$ as $c_{i}$ for $i=1,2,3 \ldots n$. Next the vertex $u_{i}$ is assigned the colour $c_{i}$ for $i=1,2$ and $c_{n+i-2}$ for $3 \leq i \leq n$. Such a colouring excludes the newly introduced vertices. Assign the colour $c_{2 n-2}$ to $v_{1,1}$ and $u_{1,2}$. Now $c_{n+1}$ to $v_{2,2}$ and $c_{2}$ to $u_{n, 1}$. All the remaining vertices are coloured $c_{1}$. Then clearly the above said colouring is acyclic, also the colouring is minimum. Because if we replace any colour which is minimum in number by a colour already used, the resulting colouring will be improper or cyclic.

Therefore $a\left[C\left(S_{n}\right)\right]=2 n-2$.

\section{Example}

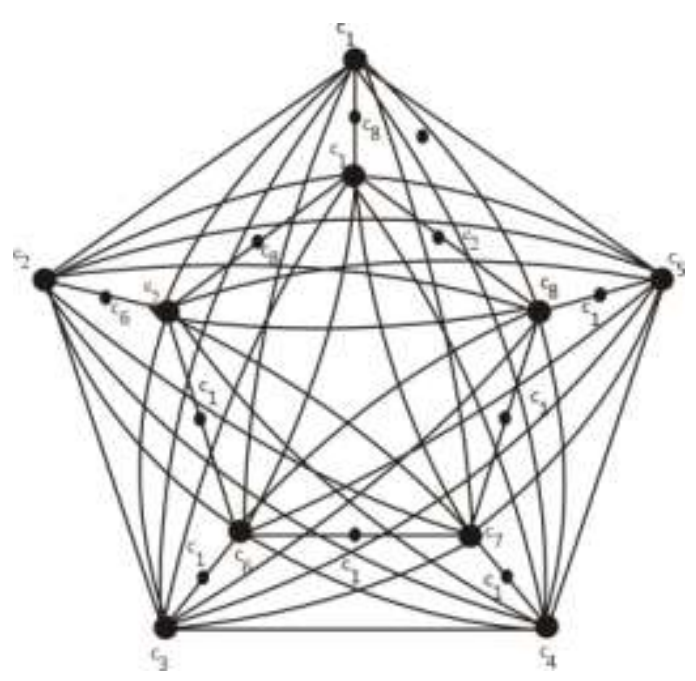

Figure 2

$a\left[C\left(S_{5}\right)\right]=8$

\section{ACYCLIC COLOURING OF $S_{n}^{o}$}

\subsection{Theorem}

The acyclic chromatic number for the Crown graph is $a\left[\left(S_{n}^{o}\right)\right]=n$ for every $n=3,4,5 \ldots$

\section{Proof}

Cosider a crown graph $G=S_{n}^{o}$ having $2 n$ vertices.Let the vertices be $\left\{u_{1}, u_{2}, u_{3} \ldots . u_{n}, v_{1}, v_{2}, v_{3}, \ldots v_{n}\right\}$.Consider the colour class $C=\left\{c_{1}, c_{2}, c_{3} \ldots c_{n}\right\}$. Assign the colour $c_{i}$ to the vertices $u_{i}$ and $v_{j}$ for $i=1,2,3 \ldots n$. This colouring is clearly acyclic.because the subgraph induced by $\left\langle c_{i}, c_{j}\right\rangle$ for all $i \neq j$ is the union of two paths $P_{1}$ always.thus the colouring is acyclic. Also this colouring is minimum. Because if we replace any colour class by another used colour class the colouring will become improper. 
Thus $\quad a\left[\left(S_{n}^{o}\right)\right]=n \quad$ for every $n=3,4,5 \ldots$

\section{Example}

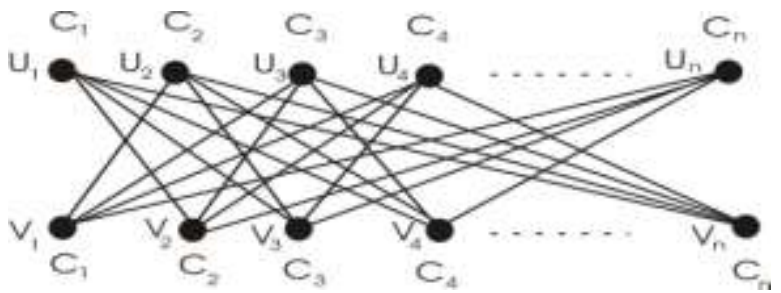

Figure 3

$$
a\left[\left(S_{n}^{o}\right)\right]=n
$$

\section{REFERENCES}

[1] N. Alon, C. McDiarmid, and B. Reed. "Acyclic colourings of graphs". Random Structures and Algorithms, 2, 277 288, 1990.

[2] J.A. Bondy and U.S.R. Murty, Graph theory with Applications . MacMillan, London, 976.

[3] O. V. Borodin "On acyclic colorings of planar graphs", Discrete Math. 25, 211-236, 1979.

[4] C. B. Boyer, A history of mathematics. New York, Wiley. 1968.

[5] A. E. Brouwer, A. M. Cohen and A. Neumaier, DistanceRegular Graphs. New York: Springer-Verlag, 1989.

[6] Danuta Michalak, On middle and total graphs with coarseness number equal 1, Spinger Verlag Graph Theory, Lagow (1981) proceedings, Berlin heidelberg, New York, Tokyo, pp. 139-150.

[7] Douglas B .West, Introduction To Graph Theory, Second Edition, Prentice-Hall of India Private Limited, New Delhi(2006).
[8] H. Eves, An Introduction to the History of Mathematics. New York: CBS College. 1983.

[9] Frank Harrary, Graph theory, Narosa Publishing House(2001).

[10] Frank Harary, Gray Chartrand, Ping Zhang, Geodetics sets in graphs. Discuss math. Graph Theory 20(2000)pp.129138.

[11] D. Frank Hsu, Harmonious Labelling of Windmill Graphs and Related Graphs, Journal of Graph Theory, Vol. 6 (1982), pp. 85-87.

[12] Graph Colouring, Wikipedia, the free Encyclopedia.

[13] B. Grünbaum. “Acyclic colorings of planar graphs”. Israel J. Math., 14(3), 390-408, 1973.

[14] Joseph .A. Gallian, “A Dynamic Survey of Graph Labeling”, The electronic journal of combinatorics, 16,11, 2009.

[15] K. Thilagavathi, K.P. Thilagavathy and N. Roopesh, "The Achromatic colouring of graphs", Electronic notes in Discrete mathematics, 153-156, 33, 2009.

[16] K. Thilagavathi and Vernold Vivin.J, "Harmonious Colouring of Total graphs, n-Leaf, Central Graphs and circumdetic Graphs" $\mathrm{PhD}$ Thesis, Kongunadu Arts and Science college, Coimbatore.

[17] K.Thilagavathi and Vernold Vivin.J and Akbar Ali.M.M, "On Harmonious colouring of Central graphs" Advances and Appications in Discrete Mathematics, 2, 17-33, 2009.

[18] Vivin J. Vernold, M. Venkatachalam and Ali M.M. Akbar, "A note on achromatic coloring of star graph families" 23:3 (2009), 251-255. 